\title{
Superconductivity and antiferromagnetism in the two-dimensional Hubbard model: a variational study
}

\author{
D. Eichenberger and D. Baeriswyl \\ Department of Physics, University of Fribourg, CH-1700 Fribourg, Switzerland.
}

(Dated: October 24, 2018)

\begin{abstract}
A variational ground state of the repulsive Hubbard model on a square lattice is investigated numerically for an intermediate coupling strength $(U=8 t)$ and for moderate sizes (from $6 \times 6$ to $10 \times 10$ ). Our ansatz is clearly superior to other widely used variational wave functions. The results for order parameters and correlation functions provide new insight for the antiferromagnetic state at half filling as well as strong evidence for a superconducting phase away from half filling.
\end{abstract}

PACS numbers: 71.10.Fd,74.20.Mn,74.72.-h

The Hubbard model plays a key role in the analysis of correlated electron systems, and it is widely used for describing quantum antiferromagnetism, the Mott metalinsulator transition and, ever since Anderson's suggestion [1], superconductivity in the layered cuprates. Several approximate techniques have been developed to determine the various phases of the two-dimensional Hubbard model. For very weak coupling, the perturbative Renormalization Group extracts the dominant instabilities in an unbiased way, namely antiferromagnetism at half filling and $d$-wave superconductivity for moderate doping [2, 3]. Quantum Monte Carlo simulations have been successful in extracting the antiferromagnetic correlations at half filling [4, 5], but in the presence of holes the numerical procedure is plagued by the fermionic minus sign problem [6]. This problem appears to be less severe in dynamic cluster Monte Carlo simulations, which exhibit a clear tendency towards $d$-wave superconductivity for intermediate values of $U$ [7].

Variational techniques address directly the ground state and thus offer an alternative to quantum Monte Carlo simulations, which are limited to relatively high temperatures. Previous variational wave functions include mean-field trial states from which configurations with doubly occupied sites are either completely eliminated (full Gutzwiller projection) [8, 9, 10] or at least partially suppressed [11]. Recently, more sophisticated wave functions have been proposed, which include, besides the Gutzwiller projector, non-local operators related to charge and spin densities [12, 13]. Our own variational wave function is based on the idea that for intermediate values of $U$ the best ground state is a compromise between the conflicting requirements of low potential energy (small double occupancy) and low kinetic energy (delocalization). It is known that the addition of an operator involving the kinetic energy yields an order of magnitude improvement of the ground state energy with respect to a wave function with a Gutzwiller projector alone [14]. In this letter, we show that such an additional term allows us to draw an appealing picture of the ground state, both at half filling and as a function of doping (some preliminary results have been published
[15, 16]).

In its most simple form, the 2D Hubbard model is composed of two terms, $\hat{H}=t \hat{T}+U \hat{D}$, with

$$
\hat{T}=-\sum_{\langle i, j\rangle, \sigma}\left(c_{i \sigma}^{\dagger} c_{j \sigma}+c_{j \sigma}^{\dagger} c_{i \sigma}\right) \text { and } \hat{D}=\sum_{i} n_{i \uparrow} n_{i \downarrow} .
$$

Here $c_{i \sigma}^{\dagger}$ creates an electron at site $i$ with spin $\sigma$, the summation is restricted to nearest-neighbor sites and $n_{i \sigma}=c_{i \sigma}^{\dagger} c_{i \sigma}$. We consider a square lattice with periodicantiperiodic boundary conditions and choose $U$ to be equal to the bandwidth, $U=8 t$. Our ansatz

$$
|\Psi\rangle=e^{-h \hat{T}} e^{-g \hat{D}}\left|\Psi_{0}\right\rangle
$$

is linked to a mean-field ground state $\left|\Psi_{0}\right\rangle$ with either a ( $d$-wave) superconducting or an antiferromagnetic order parameter. The operator $e^{-g \hat{D}}$ partially suppresses double occupancy for $g>0$, while $e^{-h \hat{T}}$ promotes both hole motion and kinetic exchange (close to half filling). In the limit $h \rightarrow 0$ we recover the Gutzwiller ansatz [11]. For $g \rightarrow \infty$ and $h \ll 1$ our variational problem is equivalent to that of the $t-J$ Hamiltonian with respect to a fully Gutzwiller-projected mean-field state.

The calculations for $h>0$ are carried out in momentum space where the operator $\hat{D}$ is not diagonal. Therefore a discrete Hubbard-Stratonovich transformation is applied to decouple the terms $n_{i \uparrow} n_{i \downarrow}$ in the operator $e^{-g \hat{D}}$ by introducing an Ising spin at each site. Expectation values are obtained using a Monte Carlo simulation with respect to the Ising spin configurations.

We discuss first the case of an average site occupation $n=1$ (half-filling), where the ground state is expected to exhibit long-range antiferromagnetic order. A commensurate spin-density wave characterized by a gap parameter $\Delta_{A F}$ is therefore the natural mean-field reference state, $\left|\Psi_{0}\right\rangle=|\mathrm{SDW}\rangle$. The order parameter is the staggered magnetization defined by

$$
M=\frac{1}{N} \sum_{i}(-1)^{i}\left\langle n_{i \uparrow}-n_{i \downarrow}\right\rangle,
$$

where $\mathrm{N}$ is the number of sites. In Table $\llbracket$ the results obtained by minimizing the energy expectation value with 
respect to the three variational parameters $g, h, \Delta_{A F}$ for an $8 \times 8$ lattice are compared with the unrestricted Hartree-Fock approximation $(g=h=0)$, the Gutzwiller wave function $(g>0, h=0)$ 17, a quantum Monte Carlo simulation [4] and a Projector Operator technique [18]. The gap parameter $\Delta_{A F}$ is very large for $g=h=0$ and decreases dramatically if $g$ and $h$ are optimized. We note that the gap parameter cannot be identified with an excitation gap, which in fact should vanish if a continuous symmetry is broken. The ground state energy is seen to vary appreciably as the parameters $g$ and $h$ are turned on and to be comparable to that found with other techniques. On the other hand, the order parameter is still rather large, at least in comparison to the accepted value of $M=0.614(1)$ for the 2D Heisenberg model $(U=\infty)$ [19], an upper bound for the Hubbard model.

\begin{tabular}{lccccc}
\hline \hline & $\mathrm{g}$ & $\mathrm{h}$ & $\Delta_{A F}$ & $M$ & $\mathrm{E} / \mathrm{t}$ \\
\hline VMC & 0 & 0 & $3.6(1)$ & $0.89(1)$ & $-0.466(1)$ \\
VMC & 0.69 & 0 & 1.3 & $0.86(1)$ & $-0.493(3)$ \\
VMC & $3.1(1)$ & $0.101(3)$ & $0.32(2)$ & $0.77(1)$ & $-0.514(1)$ \\
QMC & - & - & - & $0.42(1)$ & $-0.48(5)$ \\
PO & - & - & - & - & $-0.521(1)$ \\
\hline \hline
\end{tabular}

TABLE I: Variational results (VMC) for the 2D Hubbard model at half-filling $(8 \times 8$ lattice, $U=8 t)$, compared to quantum Monte Carlo simulations (QMC) and a Projector Operator approach (PO). The VMC data include the unrestricted Hartree-Fock approximation, the Gutzwiller ansatz and the present study.

In order to extract some information about superconducting correlations in the presence of antiferromagnetic long-range order, we have calculated the correlation function $F_{i j}=\left\langle C_{i}^{\dagger} C_{j}\right\rangle$, where

$$
C_{i}^{\dagger}=\sum_{j_{i}} \sigma_{j_{i}}\left(c_{i \uparrow}^{\dagger} c_{j_{i \downarrow}}^{\dagger}-c_{i \downarrow}^{\dagger} c_{j_{i} \uparrow}^{\dagger}\right)
$$

The sites $j_{i}$ are the four nearest neighbors of site $i$ and $\sigma_{j_{i}}=+1(-1)$ in $x$ - $(y-)$ direction. Thus $C_{i}^{\dagger}$ creates a singlet pair with $d$-wave symmetry centered at site $i . F_{i j}$ is found to decrease rapidly with increasing distance, as expected for a gapped system. For on-site correlations we find $F_{i i}=0.0637(1)$ for $h \neq 0$ and $F_{i i}=0.0592(1)$ for $h=0$, while the results for nearest-neighbor correlations are $F_{i j}=0.0171(1)$ for $h \neq 0$ and $F_{i j}=0.0155(1)$ for $h=0$. The superconducting correlations are therefore slightly enhanced by the parameter $h$.

We now discuss the effects of hole doping, and in particular the possibility of $d$-wave superconductivity, as suggested by Renormalization Group arguments 2, 3], previous variational calculations [8, 9, 10, 11, 12, 13] and quantum Monte Carlo simulations [7]. Our mean-field reference state is the BCS wave function with $d$-wave symmetry, $\left|\Psi_{0}\right\rangle=\mid d$ BCS $\rangle$, characterized by a gap parameter $\Delta$ describing pairing and a "chemical potential"

\begin{tabular}{ccccc}
\hline \hline $\mathrm{n}$ & $\mu$ & $\mathrm{g}$ & $\mathrm{h}$ & $\mathrm{E} / \mathrm{t}$ \\
\hline 0.8125 & $-0.4418(1)$ & $3.0(1)$ & $0.099(2)$ & $-0.849(1)$ \\
0.8400 & $-0.3972(3)$ & $3.2(1)$ & $0.103(2)$ & $-0.802(1)$ \\
0.9000 & $0.357(1)$ & $3.4(1)$ & $0.106(2)$ & $-0.697(1)$ \\
0.9375 & $0.620(1)$ & $3.7(1)$ & $0.110(2)$ & $-0.627(1)$ \\
0.9600 & $0.692(1)$ & $4.1(2)$ & $0.115(3)$ & $-0.583(1)$ \\
0.9700 & $0.743(1)$ & $4.3(2)$ & $0.116(3)$ & $-0.564(1)$ \\
\hline \hline
\end{tabular}

TABLE II: "Chemical potential", parameters $\mathrm{g}$ and $\mathrm{h}$ and total energy per site for different densities on an $8 \times 8$ lattice.

$\mu$ fixing the average electron density $n$ [15]. To reduce the statistical error in the Monte Carlo simulations and consequently the computational time, a fixed set of "Ising spin" configurations is first generated and then used to optimize the variational parameters [20, 21].

The ground state energy and the parameters $g, h, \mu$ are given in Table II for an $8 \times 8$ lattice and various densities. The chemical potential $\mu$ varies strongly with doping and increases so much for $n \rightarrow 1$ that the optimization becomes very difficult. The Gutzwiller parameter $g$ also increases rather strongly for $n \rightarrow 1$, which indicates that the system is "more localized" at half-filling than away from half-filling [16]. In contrast, the kinetic parameter $h$ does not vary appreciably.

The gap parameter $\Delta$ and the order parameter $\Phi=$ $\left|\left\langle c_{i \uparrow}^{\dagger} c_{j_{i \downarrow}}^{\dagger}\right\rangle\right|$ are shown in Fig. 1(a) as functions of the hole density $x=1-n$, for an $8 \times 8$ lattice. Both quantities have a maximum around $x=0.1$ and tend to zero around $x=0.18$. The limiting behavior for $x \rightarrow 0$ has not been established firmly, due to computational problems mentioned above, but our results are consistent with $\Delta \rightarrow 0, \Phi \rightarrow 0$. For the Gutzwiller wave function, the order parameter also exhibits a dome shape, but not so the gap parameter: $\Delta$ is found to increase monotonically for $x \rightarrow 0$, both for finite $U$ (inset of Fig. 1(a)) and for $U \rightarrow \infty[10$.

The condensation energy, $E_{\text {cond }}=E(0)-E(\Delta)$ where $\Delta$ is the optimal gap parameter, is depicted in Fig. 1 (b). It vanishes for $x>0.18$ and increases monotonically with decreasing $x$, even beyond the hole concentration where both $\Delta$ and $\Phi$ pass through a maximum. The limiting behaviour for $x \rightarrow 0$ is again unknown, but for $x=0$ antiferromagnetism prevails. The comparison with the Gutzwiller wave function (inset) indicates that the addition of the parameter $h$ strongly enhances the condensation energy [22]. It is worthwhile to add that according to calculations for small clusters 14] the difference $\Delta E=E_{v a r}-E_{0}$ between the variational energy $E_{v a r}$ and the exact ground state energy $E_{0}$ is of the same order for $h>0$ as the condensation energy $E_{\text {cond }}(\Delta E \approx 0.007 t$, $E_{\text {cond }} \approx 0.005 t$ at $\left.n=0.9\right)$, in contrast to the case $h=0$ where $\Delta E \gg E_{\text {cond }}\left(\Delta E \approx 0.08 t, E_{\text {cond }} \approx 0.001 t\right)$.

An important question is to what extent an $8 \times 8$ lattice is able to mimic the thermodynamic limit. Therefore we 


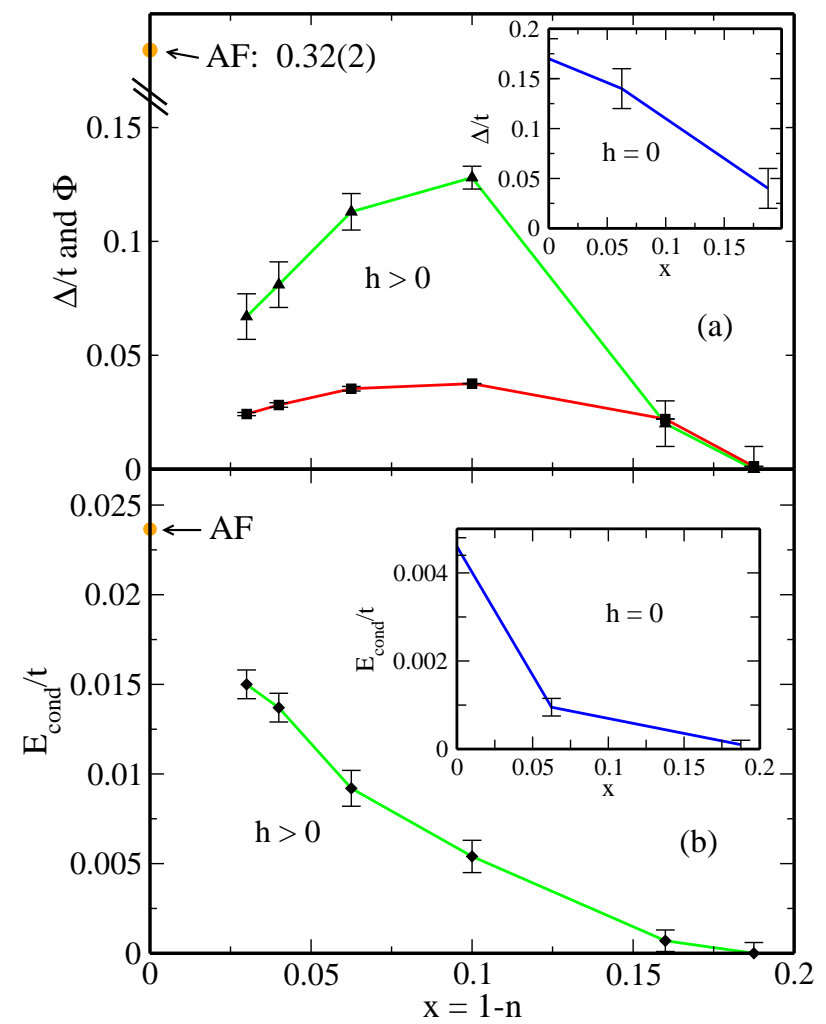

FIG. 1: (Color online) (a): Gap (triangles) and order parameter (squares) as functions of the doping for an $8 \times 8$ lattice. The mark at half-filling is the antiferromagnetic gap. The inset shows the gap parameter for the Gutzwiller wave function. (b): Condensation energy per site. (a)+(b): Error bars indicate statistical uncertainties.

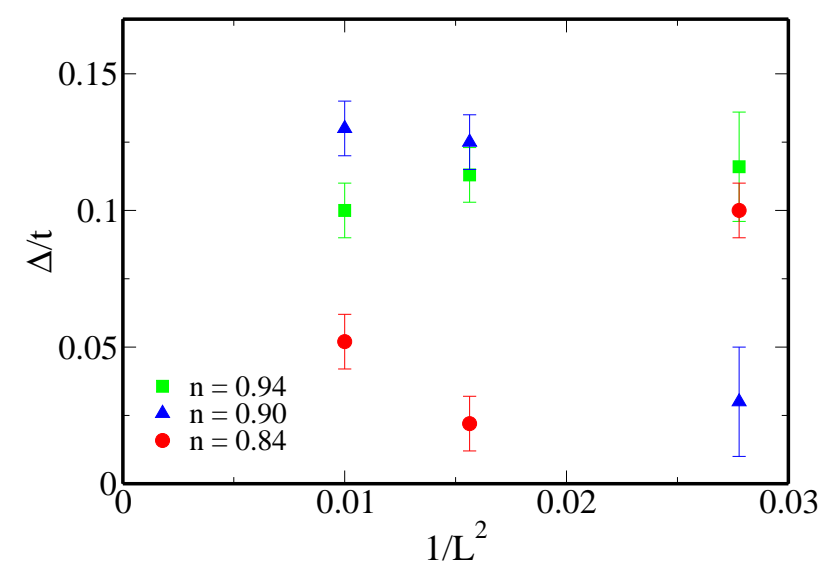

FIG. 2: (Color online) Finite-size scaling of the gap parameter for densities $n=0.84$ (circles), $n=0.90$ (triangles) and $n=$ 0.9375 (squares).

have also studied other lattice sizes. The results for the gap parameter (Fig. 2) show that the size effects are more important in regions where the gap is small $(n=0.84)$ than well inside the superconducting dome $(n=0.90$ or

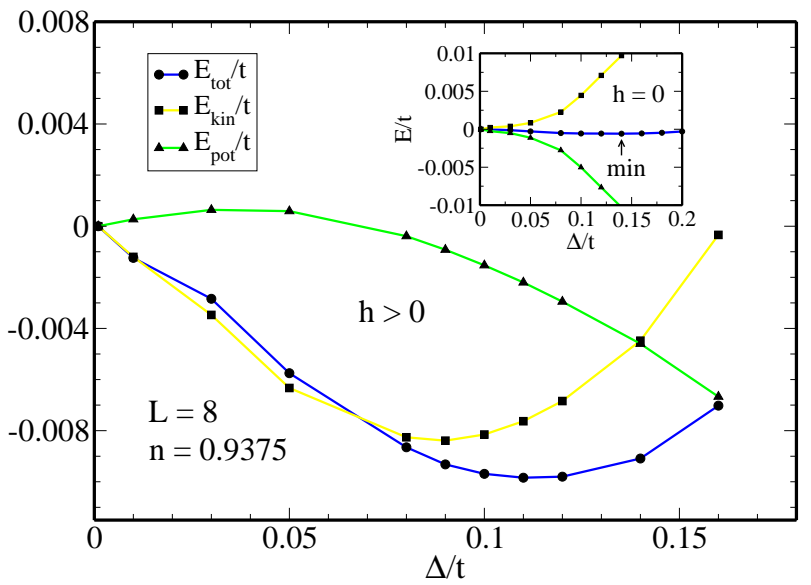

FIG. 3: (Color online) Total (circles), kinetic (squares) and potential (triangles) energies per site as functions of the gap parameter on an $8 \times 8$ lattice, for the density $n=0.9375$. For each curve $E(\Delta=0)$ has been subtracted. The relative error is smaller than the symbol size. The corresponding results for the Gutzwiller wave function are given in the inset.

$n=0.9375)$. However, even at $n=0.90$ where the gap is maximal, a $6 \times 6$ lattice is not large enough to give a reliable estimate for the thermodynamic limit.

In Fig. 3 the kinetic and the potential energies are plotted separately for a density $n=0.9375$ as functions of the gap parameter. It turns out that the maximum energy gain (the condensation energy) at $\Delta=0.11 t$ is to a large extent $(>75 \%)$ due to a decrease in the kinetic energy, in contrast to the BCS behaviour where the condensation energy is entirely due to the potential energy. Our findings are also qualitatively different from those obtained with a Gutzwiller wave function for which the kinetic energy increases monotonically with the gap parameter (inset). We have also studied the magnetic structure factor

$$
S(\mathbf{q})=\frac{1}{N} \sum_{i, j} e^{i \mathbf{q} \cdot\left(\mathbf{R}_{\mathbf{i}}-\mathbf{R}_{\mathbf{j}}\right)}\left\langle\left(n_{i \uparrow}-n_{i \downarrow}\right)\left(n_{j \uparrow}-n_{j \downarrow}\right)\right\rangle .
$$

within the superconducting phase. Fig. [4shows this function for several densities along three different lines in the Brillouin zone. The structure factor is peaked at $(\pi, \pi)$, indicating antiferromagnetic correlations. The peak decreases with increasing hole concentration. The comparison with results for $h=0$ (inset) shows that the antiferromagnetic correlations are strongly enhanced by the parameter $h$.

In summary, we have found that the addition of a "kinetic projector" $e^{-h \hat{T}}$ to the Gutzwiller wave function yields both quantitative improvements (for instance for the ground state energy or for the antiferromagnetic order parameter) and qualitative changes (such as the doping dependence of the superconducting gap or the decrease of the kinetic energy as a function of the gap parameter). Nevertheless, there remains room for im- 


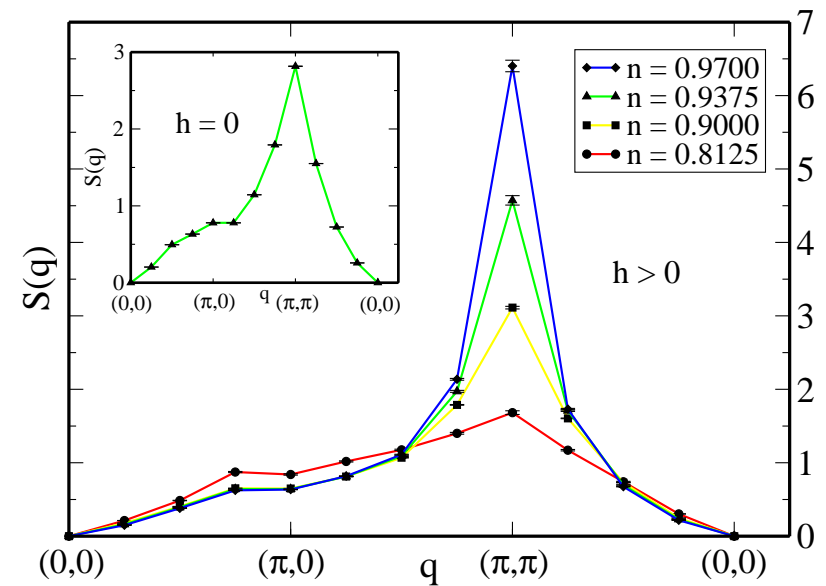

FIG. 4: (Color online) Magnetic structure factor as a function of the wave vector for different densities and an $8 \times 8$ lattice. The inset shows the magnetic structure factor for the Gutzwiller wave function at $n=0.9375$.

provement because our variational ansatz (as well as all trial states used previously) is linked to a delocalized mean-field reference state and thus requires a strong suppression of double occupancy, at least for $U=8 t$. This reflects the fact that this parameter regime corresponds to that of a doped Mott insulator which would be treated more naturally starting from a localized reference state.

Finally, we comment on the relevance of our findings for layered cuprates. The antiferromagnetic ground state for $x=0$ and a superconducting phase with $d$ wave symmetry are well established experimentally, with a slightly different doping range for superconductivity $(0.05<x<0.3$ against $0<x<0.18$ in our study $)$. The addition of a hopping term between next-nearest neighbor sites (parameter $t^{\prime}$ ) might improve this comparison. The typical size of the superconducting gap at optimal doping is $30 \mathrm{meV}$ [23], in good agreement with our result $(\Delta \approx 0.13 t \approx 40 \mathrm{meV}$ for $t=300 \mathrm{meV})$. The comparison with condensation energies extracted from specific heat measurements is less encouraging $(0.10-0.20$ $\mathrm{meV} /$ copper 24, 25] against $0.005 t=1.5 \mathrm{meV}$ in this work). Much discussion has been raised by the question of "kinetic energy driven superconductivity" where, in contrast to BCS, the energy gain arises from a decrease in kinetic energy. The reported gain of kinetic energy $\Delta E_{k i n} \approx 0.5-1.0 \mathrm{meV}$ on the basis of optical spectroscopy at optimal doping [25, 26] corresponds well to our result $\left(\Delta E_{k i n} \approx 1.1 \mathrm{meV}\right)$, but we have to be aware that the use of a low-energy cut-off in the frequency-integration of the conductivity is not unambiguous. Good agreement with experiment is also found for the strong antiferromagnetic correlations in the superconducting phase. In fact, the structure factor $S(\mathbf{q})$ determined by neutron scattering experiments shows a pronounced peak at $(\pi, \pi)$, which decreases upon doping [27.
In conclusion, the present variational calculations give an appealing picture of the ground state of the 2D Hubbard model, both at half-filling and for the doped system. Superconductivity out of purely repulsive interactions appears very naturally in this scheme. Several predictions agree surprisingly well with experiments on layered cuprates.

We are grateful for financial support from the Swiss National Science Foundation through the National Center of Competence in Research "Materials with Novel Electronic Properties-MaNEP".

[1] P. W. Anderson, Science 235, 1196 (1987).

[2] D. Zanchi and H. J. Schulz, Phys. Rev. B 61, 13609 (2000).

[3] C. J. Halboth and W. Metzner, Phys. Rev. B 61, 7364 (2000).

[4] J. E. Hirsch, Phys. Rev. B 31, 4403 (1985).

[5] S. R. White et al., Phys. Rev. B 40, 506 (1989).

[6] E. Dagotto, Rev. Mod. Phys. 66, 763 (1994).

[7] T. A. Maier, M. Jarrell, and D. J. Scalapino, Phys. Rev. B 74, 094513 (2006).

[8] C. Gros, Phys. Rev. B 38, 931 (1988).

[9] H. Yokoyama and H. Shiba, J. Phys. Soc. Jpn. 57, 2482 (1988).

[10] A. Paramekanti, M. Randeria, and N. Trivedi, Phys. Rev. B 70, 054504 (2004).

[11] T. Giamarchi and C. Lhuillier, Phys. Rev. B 43, 12943 (1991).

[12] S. Sorella et al., Phys. Rev. Lett. 88, 117002 (2002).

[13] H. Yokoyama et al., J. Phys. Soc. Jpn. 73, 1119 (2004).

[14] H. Otsuka, J. Phys. Soc. Jpn. 61, 1645 (1991).

[15] D. Eichenberger and D. Baeriswyl, Physica C (2007), doi:10.1016/j.physc.2007.03.366.

[16] D. Baeriswyl, D. Eichenberger, and B. Gut, phys. stat. sol. (b) 244, 2299 (2007).

[17] H. Yokoyama and H. Shiba, J. Phys. Soc. Jpn. 56, 3582 (1987).

[18] G. Polatsek and K. W. Becker, Phys. Rev. B 54, 1637 (1996).

[19] A. W. Sandvik, Phys. Rev. B 56, 11678 (1997).

[20] D. Ceperley, G. V. Chester, and K. H. Kalos, Phys. Rev. B 16, 3081 (1977).

[21] C. J. Umrigar, K. G. Wilson, and J. W. Wilkins, Phys. Rev. Lett. 60, 1719 (1988).

[22] It should be mentioned that the calculations for $h>0$ have been carried out for a "grand canonical ensemble" (where $\mu$ is fixed by the average density), whereas the results for $h=0$ have been obtained for a "canonical ensemble" (where the particle number is fixed and $\mu$ is a variational parameter). The two ensembles are equivalent in the thermodynamic limit.

[23] A. Damascelli, Z. Hussain, and Z.-X. Shen, Rev. Mod. Phys. 75, 473 (2003).

[24] J. W. Loram et al., Physica C 341-348, 831 (2000).

[25] E. van Heumen et al., Phys. Rev. B 75, 054522 (2007).

[26] H. J. A. Molegraaf et al., Science 295, 2239 (2002).

[27] H. F. Fong et al., Phys. Rev. B 61, 14773 (2000). 